\title{
A hierarchical multi-scale method to simulate reactive-diffusive transport in porous media
}

\author{
* A. Rai ${ }^{a}$, M. Warrier ${ }^{b}$, R. Schneider ${ }^{a}$ \\ ${ }^{a}$ Max-Planck-Institut für Plasmaphysik, D-17491 Greifswald, Germany \\ ${ }^{b}$ Institute for Plasma Research, BHAT, Gandhinagar, Gujarat, India - 382428 \\ * Corresponding author e-mail: Abha.Rai@ipp.mpg.de \\ Phone no: +49-3834-882400 \\ Fax: $+49-3834-882409$ \\ Address: HGF-NWG, Max-Planck-Institut für Plasmaphysik, Wendelsteinstrasse 1, D- \\ 17491 Greifswald, Germany.
}

\begin{abstract}
A time-dependent, hierarchical multi-scale model to simulate reactive-diffusive transport in a 3-dimensional, porous structure, using a combination of molecular dynamics (MD), kinetic Monte Carlo (KMC) and Monte Carlo diffusion (MCD) methods has been developed. The parameters that are passed from the models at the lower spatial and temporal scales (MD,KMC) to those at the higher scales (KMC, MCD respectively) are identified. The advantages and disadvantages of the different methods are discussed with respect to reactive-diffusive transport simulations of atoms in porous $3 \mathrm{D}$ structures. The model is illustrated by simulating hydrogen transport, reactions, inventory and re-emission from a porous hydrocarbon layer.
\end{abstract}


PACS codes: 52.65.Pp, 51.20.+d, 52.40.Hf, 52.65.Yy

keywords: Multi-scale Modeling, diffusion in porous media, hydrogen inventory, graphite, Monte-Carlo diffusion, kinetic Monte-Carlo, molecular dynamics, reactive-diffusive transport, Tore-Supra.

\section{Introduction}

Study and quantification of reactive diffusive transport of atoms in a porous media has innumerable applications like:

- hydrogen recycling and inventory in fusion plasma facing materials [1],

- hydrogen storage for fuel cells [2],

- diffusion along grain boundaries and defects by atomic species [3],

- tritium recovery from porous ceramic pebbles in a solid breeder reactor blanket [4],

- growth of atoms deposited on a substrate [5], etc.

In order to understand the reactive-diffusive transport of atoms in a porous media, and to quantify it, a multi-scale model has been developed [6-11]. The model is a hierarchical multi-scale model, wherein simulations at the lower scales or experimental results wherever available are used as inputs to the simulations at higher scales. It is a very general method and is widely applicable for atomic and molecular reaction-diffusion in porous geometries. The simulation methods used are molecular dynamics (MD) $[12,13]$ at the atomic scales (few nanometers in length, hundred pico-seconds in time and around 
a thousand atoms), Kinetic Monte-Carlo (KMC) [14] at the meso-scales (few tens of microns in length, and time scales from picoseconds to a few seconds for millions of diffusing particles) and a combination of KMC and Monte-Carlo Diffusion (MCD) at the macro-scales [15]. We demonstrate a method to couple KMC and MCD as part of our macro-scale simulations.

In the next section a multi-scale model for reactive-diffusive transport in porous geometry will be presented. In sections 3-6 various computational aspects in implementing the model are presented with an example of reactive-diffusive transport and inventory of hydrogen in tokamak co-deposits. Finally we present the summary and conclusions.

\section{The multi-scale model}

The multi-scale scheme developed belongs to a class of multi-scale methods known as heterogeneous multi-scale methods [16], wherein different simulation methods are used at different scales. The larger scales take inputs from modeling at the lower scales and also from experiments. The scheme is illustrated in Fig. 1. At the bottom left side of Fig. 1, PorousGeometry represents an algorithm to create a porous medium of specified void-fraction and sub-structure sizes using a statistical distribution for the sub-structure size (Section 3). The substructures can also be given an orientation. Using this algorithm granules separated by voids or randomly oriented crystallites separated by micro-voids can be generated. 
At the microscopic scales, molecular dynamics (MD) is used to simulate atomic diffusion in crystallites. The length scale for this process is a fraction of a nanometer and the time scale is a few hundred picoseconds and is limited to a few hundreds of atoms. Reactivediffusive atomic transport is represented as a thermally activated diffusive process with characteristic jump attempt frequencies $\left(\omega_{o}\right)$, migration energies $\left(E_{m}\right)$ and jump lengths $(L)$. The minimum distance of approach of two diffusing atoms which ensures molecule formation, $R_{o}$, is used to parametrize reactions. This is known as the Smoluchowski boundary condition $[17,18]$. In Section. 4.3 we will illustrate the method of parametrizing diffusion such that the parameters can be used to scale up in a Kinetic Monte-Carlo (KMC) ansatz.

Due to limitations in number of atoms that can be simulated using MD, we use Kinetic Monte-Carlo (KMC) $[14,19]$ to simulate reactive-diffusive transport in a granule. In order to scale up and model trans-granular diffusion (TGD), the internal structure of the granule must be taken into consideration. The granule consists of randomly oriented crystallites separated by micro-voids. The Kinetic Monte Carlo (KMC) scheme, (represented by DiG_TGD in Fig. 1), uses

- the parametrized MD simulation results $\left(\omega_{o}, E_{m}, L\right.$ and $\left.R_{o}\right)$ at the microscale to simulate the transport inside randomly oriented crystallites,

- experimental results for thermally activated processes at the microvoid-crystallite interfaces (MCI) and for recombination and dissociation reactions. 
- simple line of sight transport for atoms within the voids.

KMC is best suited to model reactive-diffusive transport with diverse migration energies corresponding to different processes. This yields the trans-granular-diffusion coefficient $D_{T G D}$ and the rates of various reactions $R_{T G D}$. The mesoscale simulation is described in Section 5.2 where hydrogen transport and inventory in porous hydrocarbon deposits found in fusion machines is studied.

$D_{T G D}$ and $R_{T G D}$, from the mesoscale simulations is used to implement reaction-diffusion in the granules using a Monte-Carlo diffusion (MCD) ansatz [15] at the macro-scales. KMC is used to model surface diffusion, adsorption-desorption, trapping-detrapping, dissociation and recombination at the void-granule interface (VGI). A novel method of coupled MCD in granules, and KMC in voids and the VGI, is implemented at the macroscales (DiG_Bulk in Fig. 1) for the macroscale simulations. This is described in Section 6.1.

\section{Algorithm used to produce the $3 \mathrm{D}$ porous structure}

An algorithm to generate a 3-D rectilinear parallelepiped with a specified void fraction and sub-structures (granules/crystallites) separated by gaps (voids/micro-voids) is presented. The algorithm allows specification of a mean size, random orientation (to model crystallites) and a degree of smoothness for the sub-structures.

The idea behind the algorithm is inspired by the one presented by Graziani [20] for 
creating mixtures of alloys. A porous structure can be described by specifying regions of the volume that are occupied by sub-structures (granules or crystallites) and gaps (voids or micro-voids). The volume is broken down into equal sized cubes (hitherto called cells with dimension $C L_{x}, C L_{y}$ and $C L_{z}$ in $\mathrm{X}, \mathrm{Y}$ and $\mathrm{Z}$ directions respectively). The cell size must be chosen such that it is much smaller than the smallest structural detail that needs to be simulated. Each cell is assigned a value, OccFlag equal to 0, 1, or 2 depending on whether it lies in a void, bulk or surface respectively. The Poisson distribution is used to specify the mean size of a sub-structure that has a random shape and a mean width. The smoothness of the sub-structure is adjusted by accepting only those values from the sampled Poisson distribution for adjacent planes, that lie within a certain acceptable length difference. If the sub-structures are crystallites, orientations are specified using Euler angles [21].

Fig. 2 shows a porous structure of a micron sized cube created with the above scheme using a mean element-size of $10^{-7}$ meters and a mean cell-size of $5 \times 10^{-8}$ meters for a void fraction of $10 \%$. The two perpendicular planes cutting across the volume is to give an idea of the internal structure with the differently shaded regions on the planes signifying unoccupied cells (micro-voids in this case).

In the above description of the porous geometry, it is trivial to identify the cell in which a particle is present from the $\mathrm{X}, \mathrm{Y}, \mathrm{Z}$ co-ordinates of the particle: Let $i, j$, and $k$, be the cell indices that can take values from 0 to $n_{x}-1,0$ to $n_{y}-1$, and from 0 to $n_{z}-1$ respectively. The cell indices $i, j, k$, for a particle at $\left(r_{x}, r_{y}, r_{z}\right)$ is given by the greatest in- 
teger less than or equal to $r_{x} / C L_{x}, r_{y} / C L_{y}$, and $r_{z} / C L_{z}$, respectively. The cell numbering convention followed is such that the $x$ index $i$, goes from 0 to $n_{x}-1$, for each $y$ index $j$, which goes from 0 to $n_{y}-1$. This numbering in the $\mathrm{X}-\mathrm{Y}$ plane continues for each $z$ index $k$, which goes from 0 to $n_{z}-1$. In such a numbering scheme, a particle with cell indices $i$, $j, k$, has a cell number $n_{x} \times n_{y} \times k+n_{x} \times j+i$. The cell number gives information about the local structure (occupied, unoccupied and orientations), which decides the transport behavior of the particle.

Using such a method to describe a porous structure with volume $L_{x} \times L_{y} \times L_{z}$, the region (voids, bulk or surface) where the particle resides is quickly obtained. The RAM requirement when using the above description scales as $L_{x} / C L_{x} \times L_{y} / C L_{y} \times L_{z} / C L_{z}$ since the geometry details have to be stored in the RAM for quick access. The volume simulated must be chosen such that it is sufficient to represent a sample bulk and the cell--sizes must be chosen such that the smallest relevant microscopic structural detail is simulated. This requirement can at times lead to maximum RAM requirements of 500 GB for some graphite structures and such a description of structure needs to be optimized; in many cases however, realistic simulations which lead to new physics insights can be carried out with 1-2 GB RAM on a single PC with the above description of structure.

We now describe the various methods used in the multi-scale model using hydrogen diffusion and inventory in porous co-deposited layers of tokamaks as an example. The deposits on the leading edge of the neutralizer plate of Tore Supra were analyzed [22] 
using adsorption isotherm measurements and electron microscopy. It was found that the neutralizer deposits show an ovoid shape structure. They consisted of small graphite like crystallites of size $2-4 \mathrm{~nm}$ and $7-9 \mathrm{~nm}$ parallel and perpendicular to the graphene planes respectively. The porosity of these deposits was multi-scale in nature consisting of micropores (lower than $2 \mathrm{~nm}, \sim 11 \%$ ), mesopores $(2$ and $50 \mathrm{~nm}, \sim 5 \%$ ) and macropores (more than $50 \mathrm{~nm}$ ).

\section{Molecular dynamics simulations at the micro-scales}

Molecular dynamics (MD) $[12,13]$ is used to study atomic transport in crystalline materials. As a first approximation, we use the Brenner potential [23] with Nordlund's extension [24] to simulate hydrogen isotope diffusion in crystalline graphite.

\subsection{Setting up the simulation}

The crystalline graphite samples are prepared by initializing 960 carbon atoms at the graphite crystal locations with 10 unit cells along the $\mathbf{X}$ direction, 12 along the $\mathbf{Y}$ direction and 2 in the $\mathbf{Z}$ direction of the graphite crystal. This results in 4 graphene layers as shown in Fig. 3 (the graphene layer lies along the $\mathbf{X}-\mathbf{Y}$ plane with the $\mathbf{Z}$ direction being perpendicular to the graphene layers). The initial velocities are set by using Gaussian

distributed random numbers multiplied by a mean square velocity given by $\sqrt{2 k_{B} T / m}$ in each of the three directions and making sure that the system has total momentum equal to zero. The temperature and pressure are maintained by the Berendsen temperature and 
pressure control [25]. We carry out simulations at zero pressure at the temperatures of interest so that the atomic positions relax to their minimum potential energy states consistent with the interaction potential used. A single hydrogen atom having zero velocity is introduced in between the graphene layers of a graphite crystal so produced. Periodic boundary conditions in the three directions are applied. The temperature of graphite was maintained at $150 K, 300 K, 450 K, 600 K, 750 K$ and $900 K$ and the trajectory of the interstitial hydrogen atom was written out for analysis of the diffusion co-efficient as described in $[6]$.

\subsection{Observations}

We observe that the interstitial does not show any cross graphene layer diffusion at any of the graphite temperatures simulated. The position of the interstitial is output at every time step of the MD code and the trajectory is analyzed to verify the diffusive nature of the hydrogen isotope transport. Fig. 4 shows hydrogen interstitial trajectories (in yellow) at $150 K$ and $900 K$ of the graphite crystal. The view is along the $\mathbf{Z}$ axis and all atoms having the same color are carbon atoms belonging to the same graphene layer. One can easily identify the hexagonal structures typical of the graphene layer. Diffusion with a short step-size is seen at all target temperatures, with a long step-size diffusion making its appearance at higher temperatures $(\geq 450 K)$.

The hydrogen isotope equilibrates with the graphite crystal in a couple of picoseconds. It moves around in the potential well of the vibrating carbon atoms and makes a jump from 
one potential well to another depending on the migration energy barrier and the graphite temperature. It is seen that typically 100 such jump events occur during the course of a 100 picoseconds simulation at $150 K$ and more than 400 jumps occur at $900 K$. This is sufficient to provide equilibration and statistics to analyze the interstitial diffusion in the graphite crystal.

\subsection{Scaling up to meso-scales}

Analysis of the diffusion must be done from a point of view of scaling up in time, length and number of particles simulated. There exist various methods to overcome the limitations of MD (see [26] for a review of these methods). Molecular dynamics gives a microscopic insight into the physical process of diffusion of a hydrogen isotope in crystalline graphite. The hydrogen isotope vibrates in a potential well formed by the vibrating carbon atoms and wanders in the $\mathrm{X}-\mathrm{Y}$ plane which is the plane parallel to the graphene layers (Fig. 4). During the vibrations it exchanges energy with the vibrating carbon atoms around it. At some point in the simulation, due to interaction with its neighboring atoms, it gains enough energy to exit the potential well it is trapped in and enters a new potential well at a new location within the graphite crystal. Most of the simulation time is spent in these vibrations in a potential well. Note that the hydrogen isotope also loses information about its entry pathway into the potential well within a few vibrations. Its exit route from the potential well is independent of its entry path. This implies that each consecutive jump is independent of its past history. Therefore if we can reformulate the above diffusion phenomena as probabilities for jumping out of the potential traps, with probable lengths 
and directions for the jumps, we can scale up a lot in the time and in the number of particles simulated using Kinetic Monte Carlo (KMC) [14] described in the next section.

We obtain the diffusion coefficient of the hydrogen isotope by using the Einstein relation:

$$
D=\lim _{t \rightarrow \infty} \frac{1}{2 d t}[r(t)-r(0)]^{2}
$$

where $D$ is the diffusion coefficient, $d$ is the dimensionality of the system and $\langle r(t)\rangle$ is the position of the atom at time $t$. This is proportional to the slope of the mean square displacement of a single particle undergoing Brownian motion at the long time limit and is also known as the "tracer diffusion coefficient" [27].

Specifically what is needed for scaling up is to characterize the diffusion in terms of the jump attempt frequency $\omega_{o}$, the migration energy $E_{m}$, and the jump distance $L$.

$$
\omega=\omega_{o} e^{\frac{-E_{m}}{k_{b} T}}
$$

where, $\omega$ is the jump frequency (the frequency with which the interstitial jumps a distance $L$ in a specified direction), and $k_{B}$ is the Boltzmann's constant. Such a characterization assumes that the diffusion process is Arrhenius like

$$
D=D_{o} e^{\frac{-E_{m}}{k_{b} T}}
$$

with $D_{o}=w_{o} \times L^{2}$.

Analysis of the hydrogen isotope trajectories as described in [6] suggest the existence of two different diffusive processes, one with a low $\omega_{o}=6.8 \times 10^{12} \mathrm{~s}^{-1}$, that varies as the 
inverse square root of isotope mass, and small $E_{m}=0.015 \mathrm{eV}$ with short jump lengths $(L \simeq 2.5-3 \AA)$, and the other with a higher $\omega_{o}=2.7 \times 10^{13} s^{-1}$, independent of the isotope mass, larger $E_{m}=0.27 \mathrm{eV}$ and longer jump lengths $(L \simeq 10 \AA)$. Note that such a parametrization fits well with our observations of short and long jumps in the MD simulation.

\section{Meso-scale simulations using Kinetic Monte Carlo}

Fichthorn and Weinberg [14] established that Monte Carlo simulations can be used to simulate the dynamics of a Poisson process if

- Transition probabilities between different states of the system are created such that a dynamical hierarchy of transition rates to correctly model the microscopic barrier crossings (events) is established;

- Detailed balance is satisfied at equilibrium, which means that all the equally probable eigen states of a system at equilibrium can be accessed with the same probability;

- The various events are independent, and

- Time increments are calculated appropriately as defined by Eqn.4.

$$
\Delta t=-\frac{\ln (U)}{\sum_{i} n_{i} r_{i}}
$$

where, $n_{i}$ is the number of atoms capable of undergoing a transition labeled $i, U$ is a uniform random number between zero and one, and $r_{i}$ the transition rate. 
The time calculated in this manner corresponds to the real time evolution of the system. In principle the time evolution of the system can be followed self-consistently. But computationally this is efficient only when the different possible thermally activated processes do not have vastly different migration energies.

\subsection{The Bortz-Kalos-Lebowitz algorithm}

All thermally activated processes taking place in the system are characterized by their migration energy, $E_{m}$. The number of detrapping events from the trap follows an Arrheniuslike relation (Eqn. 2). Let there be j possible jumps for a particle belonging to the species i. let $\omega_{i}^{j}$ be the jump rate of the $j^{\text {th }}$ jump and $n_{i}$ be the number of particles, then one can define a cumulative rate, $R_{i}$, where

$$
R_{i}=\sum_{j=0}^{N J u m p s} n_{i} \omega_{i}^{j}
$$

In the Bortz-Kalos-Lebowitz (BKL) algorithm [19,28]during each simulation step a particle is picked with a probability proportional to its jump rate using the following procedure:

(1) Initialize the system at $t=0$

(2) Form a list of all the rates $r_{j}$ of all possible transitions (jumps) in the system 
(3) Calculate the cumulative rate:

$$
R=\sum_{i=0}^{N \text { Species }} R_{i}=\sum_{i=0}^{N \text { Species NJumps }} \sum_{j=0} n_{i} \omega_{i}^{j}
$$

(4) Get a uniform random number $U \in[0,1]$.

(5) Find the event to carry out, $i$ by finding the $i$ for which

$$
R_{i-1}<U R<R_{i}
$$

(6) Carry out event $i$.

(7) Check for reactions and update sources and sinks.

(8) Get a new uniform random number $U \in[0,1]$

(9) Update the time with $t=t+\Delta t$ where

$$
\Delta t=-\frac{\ln (U)}{R}
$$

and update the KMC step count

(10) If maximum number of KMC steps is not reached go to step (2), else exit.

The above algorithm allows us to simulate reaction-diffusion of millions of particles diffusing in a porous geometry. We initialize the atomic positions (step-1). Step 2-5 help in picking an atom with a probability proportional to its jump rate. Step 6 involves implementing what happens to an atom once it is detrapped. For example in the simulated MD problem of diffusion in a graphite crystal, the detrapped atom can move in a ran- 
dom direction in the 2D plane of the crystallite by a distance equal to its jump length. Step 7 involves implementation of the Smoluchowski boundary condition [18] to simulate recombination. This is achieved by checking the distance between the atoms. If the distance between two atoms gets smaller than a certain distance $\mathrm{r}_{o}$ they recombine to form a molecule. This is equivalent to having a step potential well for interaction of the species at a radial distance $\mathrm{r}_{o}$. Such boundary condition is valid for the cases with strong binding energies (like hydrogen recombination or hydrogen trapped at the open bond site) where reflection becomes a rare event once the atom gets closer than a cutoff distance [17]. Step 9 updates the time and step 10 is a check for stopping the simulation. Using the above algorithm, we have verified the parametrization of the MD simulations and also with analytic formulas in the pure diffusion limit $[6,8]$.

We want to implement a continuous influx of hydrogen atoms from the plasma incident on the co-deposits. The average number of atoms introduced per second is: $\left(S \times \Gamma \times L_{x} \times L_{y}\right)$, where $\mathrm{S}$ is the sticking coefficient of the incoming atoms for the given surface, $\Gamma$ is the incident flux and $L_{x} \times L_{y}$ is the surface area of the sample. All thermally activated processes and the incident flux of atoms too can be viewed as Poisson process and the BKL algorithm can be used to decide which event will happen in the given $\Delta t$. Particles are introduced in the system according to a Gaussian distribution with a range of penetration of $7.5 \times 10^{-9} \mathrm{~m}$ and a standard deviation of $6.0 \times 10^{-9} \mathrm{~m}$ along the $\mathrm{z}^{-}$-direction. For high fluxes seen in fusion plasma facing materials $\left(10^{20}-10^{24} \mathrm{H} \mathrm{m}^{-2} \mathrm{~s}^{-1}\right)$ a lot of particles are introduced into the system using this ansatz. This leads to a high cumulative rate and eventually very small $\Delta t$ (Eq. 4). High migration energy processes like detrapping does 
not occur and it becomes very difficult to sample the high migration energy events within the possible simulation times. We therefore do not treat the incident flux of particles as a Poisson process within the BKL Algorithm. Based on the thermally activated events we calculate the cumulative rate and find the $\Delta t$ and introduce $\Delta N$ particles in the simulation given by

$$
\Delta N=S \times \Gamma \times \Delta t \times L_{x} \times L_{y}
$$

A quasi steady-state is said to be achieved when the incoming flux of the ions is equal to the outgoing flux.

The above scheme used to make the problem computationally tractable has one disadvantage. For problems that have a wide variation in the activation energies, the higher activation energy events can occur when the species that contribute to the low-activation energy events become absent. Occurrence of such high activation energy events (rare events) leads to a large value for $\Delta t$. This implies that the number of particles introduced during the occurrence of a rare event is unrealistically large and the problem may not be computationally tractable due to large RAM (Random Access Memory) requirements. Introducing a large number of particles is also unrealistic because low activation energy events can now take place preferentially even if a few particles are introduced. To overcome this problem, the total number of particles that are introduced during the rare event case is distributed over many steps (e. g. 100 particles per simulation step). In this work we have only diagnosed quasi steady-state quantities where the measured quantities are 
practically constant over a period of time. The number of particles introduced in such cases must be varied to verify the repeatability of results.

\subsection{Example of KMC simulation at the mesoscales}

Based on the experimental structural analysis [22], the hydrogen retention and re-emission of the deposits found on the leading edge of the neutralizer (named N-LE) of Tore Supra is simulated. The typical plasma flux near this region is about $10^{16}-10^{17} \mathrm{D}^{+} \mathrm{cm}^{-2} \mathrm{~s}^{-1}$ and the temperature can reach up to $1500 \mathrm{~K}$. The incident ion energy near the neutralizer region is typically $50-300 \mathrm{eV}$.

In order to scale up, we must now include the realistic structure of porous graphite. Additional physics mechanisms like trapping/detrapping at traps, recombination, dissociation, etc of the hydrogen isotopes must be added to extend the model to simulate TGD of hydrogen isotopes. The input parameters have been taken from molecular dynamics simulations and experiments. The following processes have been included into the model for a hydrogen atom inside a granule of graphite.

- Diffusion within the crystallites: short $\left(0.015 \mathrm{eV}, 0.38 \mathrm{~nm}, 6.8 \cdot 10^{12} \mathrm{~s}^{-1}\right)$ or long $(0.27 \mathrm{eV}$, $\left.0.1 \mathrm{~nm}, 2.74 \cdot 10^{13} \mathrm{~s}^{-1}\right)$ jumps $[6,10]$.

- Surface diffusion $\left(0.9 \mathrm{eV}, 3.46 \mathrm{~nm}, 1.0 \cdot 10^{13} \mathrm{~s}^{-1}\right)[1,7,29]$

- Trapping (based on predefined trap site distribution or trapping probability)

- Detrapping $\left(2.6 \mathrm{eV}, 0.3 \mathrm{~nm}, 1.0 \cdot 10^{13} \mathrm{~s}^{-1}\right)[1]$

- Going into the bulk $\left(2.67 \mathrm{eV}, 0.3 \mathrm{~nm}, 1.0 \cdot 10^{13} \mathrm{~s}^{-1}\right)$ [30] 
- Desorption $\left(1.91 \mathrm{eV}, 0.2 \mathrm{~nm}, 1.0 \cdot 10^{13} \mathrm{~s}^{-1}\right)[31]$

- Recombination (Not a thermally activated process. Treated using Smoluchowski boundary condition $[17,10,18])$.

A hydrogen molecule can undergo following processes

- Diffusion $\left(0.06 \mathrm{eV}, 0.2 \mathrm{~nm}, 1.0 \cdot 10^{13} \mathrm{~s}^{-1}\right)$

- Dissociation $\left(4.48 \mathrm{eV}, 0.2 \mathrm{~nm}, 1.0 \cdot 10^{13} \mathrm{~s}^{-1}\right)$

- Desorption $\left(H_{2}\right.$ is chemically inactive species, so it gets desorbed as it reaches the geometrical surface)

- Detrapping (detrapping of two trapped hydrogen atoms close enough to form molecule): $\left(2.3 \mathrm{eV}, 0.4 \mathrm{~nm}, 1.0 \cdot 10^{13} \mathrm{~s}^{-1}\right)[32]$.

\subsection{Setting up the simulations at meso-scales}

At meso-scales, the geometry was implemented in our simulations by creating a porous structure of $3 \times 10^{-7} \mathrm{~m}, 3 \times 10^{-7} \mathrm{~m}, 1 \times 10^{-7} \mathrm{~m}$ in $\mathrm{X}, \mathrm{Y}$ and $\mathrm{Z}$ direction respectively, representing a typical granule with periodic boundary conditions in $\mathrm{X}$ and $\mathrm{Y}$ direction. The basic cell size was $1 \times 10^{-9} \mathrm{~m}$. The sample was composed of micropores having $11 \%$ void fraction with void size $1 \times 10^{-9} \mathrm{~m}, 1 \times 10^{-8} \mathrm{~m}, 1 \times 10^{-8} \mathrm{~m}$ and mesopores having $5 \%$ void fraction with void size $1 \times 10^{-8} \mathrm{~m}, 1.8 \times 10^{-8} \mathrm{~m}, 1.8 \times 10^{-8} \mathrm{~m}$ in $\mathrm{X}, \mathrm{Y}$ and $\mathrm{Z}$ directions respectively. Out of the total $9 \times 10^{6}$ cells in the simulation box $\sim 21 \%$ are surface cells. We are interested in the analysis of thermalized hydrogen. The ion beam energy we have considered in our simulation is $300 \mathrm{eV}$. We approximate the profile of the ions given by 
TRIM calculations to a Gaussian distribution with a range of penetration of $7.5 \times 10^{-9} \mathrm{~m}$ and a standard deviation of $6.0 \times 10^{-9} \mathrm{~m}$ along the $\mathrm{z}$-direction. The deposits have 0.75 $\%$ active carbon sites, therefore, in our simulation, every time an atom jumps, a trapping probability of 0.0075 is used.

A 2D schematic view of such a structure is shown in Fig. 5. First the simulations are done using $\mathrm{KMC}$ at the meso-scale where only one granule containing micro-pores and mesopores is simulated. Then the diffusion coefficient and the recombination rate calculated at the meso-scales is used to scale up to macro-scales where a bunch of granules separated by macrovoids are simulated using a Monte Carlo Diffusion method described in Section 6.

\subsection{Parametrizing results at the mesoscopic length scales}

Haasz et al. [33] treated recombination as thermally activated process and have parametrized it using the classical equation for recombination

$$
\kappa_{s s}=8 \pi \Delta_{s} R_{s s} N_{c}
$$

where $\kappa_{s s}$ is the recombination rate, $\Delta_{s}$ is the surface diffusion coefficient, $N_{c}$ is the atomic density of the graphite crystallites (of the order of $10^{29} \mathrm{C} / \mathrm{m}^{3}$ ) and $R_{s s}$ is the radius of recombination. Deusing et al. [34] reported $R_{s s} \sim 0.2 \mathrm{~nm}$. In the KMC parametrization presented here recombination is not treated as a thermally activated process. The tem- 
perature dependence on recombination rates comes through the diffusion coefficient of atoms, which is temperature dependent.

It has been observed that the recombination rate depends on the internal structure of the sample and is facilitated by the higher internal porosity, along which the atoms can diffuse and eventually recombine to form molecules. Eq. 6 does not include any dependence of the recombination rate on the internal structure of the sample. In the present work the recombination process was parametrized for a given internal structure of the sample at the meso-scale and was used as an input parameter for scaling up to macro-scale.

In order to quantify the recombination rate, each particle is followed and assigned an average time. When a recombination event takes place the maximum of the times of the two recombining particles is taken $\left(t_{\text {recomb. }}\right.$, the time spent by the particle in the system before it recombines). The quantity $1 / t_{\text {recomb. }}$ gives the recombination rate for one recombination event. Similar procedure is followed for the other recombination events too and finally an average is taken of all $1 / t_{\text {recomb. }}$ 's. This quantity was used as the average recombination rate $\left(\kappa_{s s}\right)$.

Fig.6 shows the recombination rate for different incident fluxes ranging from $10^{17} \mathrm{H} / \mathrm{m}^{2} / \mathrm{s}$ (ion beam experiments) to $10^{24} \mathrm{H} / \mathrm{m}^{2} / \mathrm{s}$ (plasma fusion devices) as a function of target temperature. Note that for the lower fluxes, there is a peak recombination rate and this peak shifts to higher temperatures as the flux increases. It is also seen that on an average, 
the recombination rate increases with increasing flux. At higher incident fluxes there is no peak. At a given incident flux, the density decreases with temperature tending to decrease the recombination rate, whereas, the diffusion coefficient increases with temperature tending to increase the recombination rate. This balance is what leads to a maximum in the recombination rates as the temperature is increased [35].

\section{$6 \quad$ Scaling up to Macroscales}

A schematic for bulk diffusion in porous graphite is shown in Fig. 7. Using the algorithm described in Section.3, a typical bulk co-deposit structure with macropores is generated. The orientations of the sub-structures are switched off in this case. The incident hydrogen deposition profile, damage profile, etc., can be obtained from TRIM, TRIDYN [36,37] simulations which simulate the momentum transfer processes in a isotropic solid. At the end of the TRIM and TRIDYN simulations, the incident atoms lose their energy and thermalize with the target. Our simulations start from the point where the momentum transfer processes end. The thermalized hydrogen atom positions are initialized to have a uniform distribution along $\mathbf{X}$ and $\mathbf{Y}$. Along $\mathbf{Z}$, a Gaussian distribution with a specified standard deviation, centered at a depth $R$ (mean penetration depth) is used to distribute the hydrogen atoms (Fig. 7). The particles are then classified as belonging either to the internal surface facing a macropore or belonging to the bulk of the co-deposit, depending on their locations within the structure. 
In order to simulate a sample having macropores the system dimension should be several micro-meters. Using KMC alone in this case is very inefficient, therefore, KMC has been coupled with Monte-Carlo Diffusion (MCD). A Monte Carlo diffusion algorithm [15] (briefly described in Section. 6.1) is implemented for bulk diffusion in co-deposits. MCD method is very fast but it needs diffusion coefficients and reactions rates as input. Therefore, first, at meso-scale KMC is used to calculate diffusion coefficient $D_{T G D}$ and reaction rates within a granule (bulk co-deposit having only micropores and mesopores). This was done in Section 5. Then at macroscale this information is used as input in MCD algorithm. Fig. 8 shows the coupling scheme of KMC and MCD at macroscale. The atoms at the void-granule interface and within the voids are transported using KMC, which also provides the time step $\Delta t$ for the MCD. The diffusing particles in the voids recombine if they come closer than a minimum distance. Particles reaching the surface have a probability of being desorbed. This is also handled within the KMC algorithm. For transport and reactions within the granules MCD is used. Recombination rate depends on the incoming flux, temperature and the internal structure of the sample. The diffusion coefficient depends on the sample temperature and internal structure. Therefore the input parameters for scaling up to macro-scales are taken from the meso-scales for a given flux, temperature and the internal structure.

\subsection{Brief introduction to Monte Carlo-Diffusion simulation}

The Monte-Carlo diffusion methods are efficient when a large number of dimensions or complex geometries are involved. It is especially suitable to solve diffusion of a large 
number of particles in a complex geometry with independent jumps classified as a standard Wiener process in literature [15].

The fluid continuum diffusion equation is given by

$$
\frac{\partial n}{\partial t}=\nabla(D \nabla n)+S
$$

where $n$ is the density, $D$ is the diffusion coefficient of the particles, $t$ is the time variable, $S$ is the sources or sinks in the system during the diffusion, and $\nabla n$ stands for the density gradient. $n$ is determined by the distribution of the $N$ atoms in the simulation volume. For a known value of $\mathrm{D}$, the above diffusion can be represented as a random walk of the $N$ particles with the jump size given by

$$
\Delta r=\sqrt{2 D \Delta t} \zeta
$$

where $\Delta t$ is the time step and $\zeta$ is sampled from a random number distribution satisfying $<\zeta>=0$ and $\left\langle\zeta^{2}>=1\right.$ (we use a Gaussian distribution for $\zeta$ ). This representation is valid for $\frac{D}{\nabla D}>>\sqrt{2 D \Delta t}$. Interactions are included by increasing or decreasing the number of particles diffusing at each time step by a fraction $e^{-\frac{\Delta t}{\tau}}$ where $\tau$ is the characteristic time for the interaction to occur.

The time step $\Delta t$ calculated within the KMC scheme is used to decide the number of recombination events $\left(N_{\text {Recomb }}\right)$ that should take place in the time $\Delta t$

$$
N_{\text {Recomb }}=\kappa_{s s} \times \Delta t
$$


MCD algorithm is used to carry out particle transport and reactions (recombination) within the granules (using the diffusion coefficient and reaction rates obtained at mesoscale). The following algorithm is used to implement this concept.

(1) Input D, $\Delta t, N_{\text {Recomb }}$, NSteps (number of MCD steps)

(2) Initialize positions of the $\mathrm{N}$ particles and set $\mathrm{t}=0$;

(3) Set Nnow $=$ N.

(4) Loop over NSteps

(a) Loop over the Nnow particles

(b) Sample a Gaussian distribution, distributed around 0, with a variance $=1$, to obtain $\zeta$ and use Eqn. 8, to obtain the jump length $(\Delta r)$ of the particle.

(c) Sample points uniformly distributed on a sphere of unit radius to obtain a direction for the jump in 3D. Each sampling results in 3 components, say $d x$, dy and dz such that $d x^{2}+d y^{2}+d z^{2}=1$.

(d) Change the particle position $r x=r x+\Delta r \times d x$, $r y=r y+\Delta r \times d y$, and $r z=r z+\Delta r \times d z$

(e) Choose $2 \times N_{\text {Recomb }}$ particles randomly and carry out $N_{\text {Recomb }}$ recombination events

(f) Update the number of particles of different species

(g) End loop

(5) $\mathrm{t}=\mathrm{t}+\Delta t$

(6) End loop over NSteps 


\subsection{Setting up the simulation at macro-scales}

At macro-scales, the geometry was implemented in our simulations by creating a porous structure of $2 \times 10^{-6} \mathrm{~m}, 2 \times 10^{-6} \mathrm{~m}, 2 \times 10^{-6} \mathrm{~m}$ in $\mathrm{X}, \mathrm{Y}$ and $\mathrm{Z}$ direction respectively. The basic cell size was $1 \times 10^{-8} \mathrm{~m}$. The target is composed of macropores having $10 \%$ void fraction with void size $1 \times 10^{-7} \mathrm{~m}, 1 \times 10^{-7} \mathrm{~m}, 6 \times 10^{-7} \mathrm{~m}$. For the hydrogen atom and molecule transport, KMC was used in the voids and MCD was used in the co-deposit bulk. Periodic boundary conditions are applied along the $\mathbf{X}$ and $\mathbf{Y}$ directions, which are the directions parallel to the target surface. Along the $\mathbf{Z}$ direction, which lies perpendicular to the surface, the structure is replicated for $\mathbf{Z}>0$. The target surface lies at $Z=0$ and faces the plasma located in the negative $\mathbf{Z}$ direction. Fig. 9 shows the void size distribution in $\mathrm{X}, \mathrm{Y}$ and $\mathrm{Z}$ directions created from the simulation.

\subsection{Results of the macroscale simulation}

Fig. 10 shows the retained amount of hydrogen at meso-scales (sample having micropores and meso-pores together) and macro-scales (sample having macropores) from the simulations of typical structures corresponding to Tore Supra deposits subjected to flux of $10^{20} \mathrm{H} / \mathrm{m}^{2} / \mathrm{s}$. It must be noted that the fraction of hydrogen retained by micropores and mesopores till $1600 \mathrm{~K}$ is $\simeq 90 \%$ whereas for macropores it is around $60 \%$. Fig. 11 view of the $\mathrm{X}-\mathrm{Y}$ plane located at $\mathrm{Z}=5.5 \times 10^{-8} \mathrm{~m}$. All the atoms having their $\mathrm{Z}$-position between 50 to $60 \mathrm{~nm}$ have been projected on the $\mathrm{X}-\mathrm{Y}$ plane. It clearly shows that the 
hydrogen which is released from the mesopores get trapped at the macropore surfaces. This gives rise to an internal inventory (or deposition) on macropore surfaces. This value is in good agreement with the experiments where during long pulses, particle balance gives evidence that a constant fraction of the injected gas (typically $50 \%$ of the injected fuel) is retained in the wall for the duration of the shot, showing no sign of wall saturation after more than 6 minutes of discharge [38].

Fig. 12 shows the hydrogen release behavior for mesopores and macropores. From the total released amount the fraction contributed by atoms and molecules is plotted on the Y-axis. The retained amount for these two cases are given in Fig. 10 and one can find for each temperature the exact amount of hydrogen released from the retained amount $(100 \%$ - retained amount (\%)). At meso-scales, the hydrogen release curve follows the experimental results of [39] and the model calculation of [33], i.e. the released flux is mainly molecular hydrogen at lower temperatures and atomic hydrogen at higher temperatures. At macro-scales, all the hydrogen atoms which are distributed on the macropore surfaces, are trapped (high trapping probability of 0.0075 ) or they diffuse deep into the bulk. The hydrogen atoms which land up in the granules diffuse within it (MCD, using TGD coefficient) and get recombined to form hydrogen molecules (determined by the recombination rate calculated at meso-scales). As explained above, all the hydrogen released as atoms from the mesopores get trapped again at the macropores surfaces. The hydrogen which is released in the molecular form from the mesopores, due to its chemical inactivity is able to reach the geometrical surface and get released from there. Therefore, at macro-scales 
even at very high temperatures hydrogen is released mainly in the molecular form.

\section{Limitations and future directions}

As discussed in previous sections for a system where different possible thermally activated processes have comparable migration energies, the time can be calculated using Eq. 4 and a Global time for the system can be calculated with good statistical accuracy. Note that the probability of occurrence of an event is given by $\omega^{j}=\omega_{o}^{j} e^{\frac{-E_{m}^{j}}{k_{b} T}}$ and even small differences in the migration energy, $E_{m}$, can lead to huge difference in the occurrence probability of the events and the resulting $\Delta t$. In that case the definition of a Global time for the system where the high migration energies (or rare events) are also sampled statistically well enough needs very long computational run time. So, if one aims to follow the time evolution of the system, the inability to define a Global time for a system having very different migration energies is a major limitation of KMC in such a scenario. However other steady-state quantities can be calculated with sufficient accuracy.

As discussed before, in the present work the reactions rates calculated at the meso-scales for a given internal structure, flux and temperature were used to scale up. In future, another good approach could be to parametrize the reaction rates in terms of the local number density of the atoms and the diffusion co-efficient (the later presently includes the effect of the temperature and the internal structure). 


\section{Conclusion}

We have developed a multi-scale scheme to simulate reactive-diffusive transport in a porous media. The model reproduces experimental results for hydrogen retention and reemission in porous co-deposits in fusion machines and gives new insight into the effect of internal structure on the transport of hydrogen. New methods of scaling up the diffusion and reactions from micro-scales to meso-scales to macro-scales have been devised. To our knowledge this is the first time a KMC and a 'diffusion MC' ansatz has been combined, with the KMC deciding the time step for the simulation. An algorithm to create a porous geometry has been developed. It needs further development to be able to produce specific properties like tortuosity of the pores and the BET surface area of the porous media being modeled. We have till now scaled up in lengths and time to fusion relevant values and can also accommodate fusion relevant densities and fluxes.

\section{Acknowledgements}

Ralf Schneider and Abha Rai acknowledge funding of the work by the Initiative and Networking Fund of the Helmholtz Association. Manoj Warrier acknowledges the International Max-Planck Research school on bounded plasmas and the Max-Planck India Fellowship for support and funding during the course of this work. 


\section{References}

[1] K. L. Wilson et. al. Trapping, detrapping and release of implanted hydrogen isotopes. Atomic and plasma-material interaction data for fusion (supplement to the journal Nuclear Fusion, 1:31-50, (1991).

[2] Jr Ruy Sousa and Ernesto R. Gonzalez. Mathematical modeling of polymer electrolyte fuel cells. Jnl. of Power Science, 147:32-45, (2005).

[3] J. C. Fisher. Calculation of diffusion penetration curves for surface and grain boundary diffusion. Jnl. Appl. Phys., 22:74-77, (1951).

[4] S. Tanaka, A. Kawamoto, M. Yamawaki, T. Terai, Y. Takahashi, H. Kawamura, and M. Saito. In-situ tritium release experiments from solid breeding materials (tttex) - tritium diffusion coefficients and surface reaction on lithium aluminate. Fusion Engng. and Des., 8:155-160, (1989).

[5] A.A. Benmassaoud and R.W. Paynter. Influence of the power and pressure on the growth rate and refractive index of a-c:h thin films deposited by r.f. plasma-enhanced chemical vapour deposition. Thin Solid Films, 287:125-129, (1996).

[6] M. Warrier, R. Schneider, E. Salonen, and K. Nordlund. Modeling of the diffusion of hydrogen in porous graphite. Physica Scripta, T108:85, (2004).

[7] M. Warrier, R. Schneider, E. Salonen, and K. Nordlund. Multi-scale modelling of hydrogen isotope diffusion in graphite. Contrib. Plasma Phys., 44(1-3):307-310, (2004).

[8] M. Warrier, R. Schneider, E. Salonen, and K. Nordlund. Multi-scale modeling of hydrogen isotope transport in porous graphite. Jnl. Nucl. Mater., 337-339:580-584, (2005). 
[9] M. Warrier. Multi-scale modeling of hydrogen isotope transport in porous graphite. Ph.D thesis submitted to Ernst-Moritz-Arndt University, http://www.ipr.res.in/ manoj/, (2004).

[10] A. Rai, R. Schneider, and M. Warrier. Hydrogen release and retention from porous graphite. Jnl. Nucl. Mater., 374:304-312, (2008).

[11] A. Rai. Dynamic monte carlo modeling of hydrogen isotope reactive diffusive transport in porous graphite. Ph.D Thesis, Ernst Moritz Arndt Universitat(Greifswald, Germany), niversitat.

[12] D. Frenkel and B. Smit. Understanding molecular simulations: From algorithms to applications. Academic Press, Computational Science Series, Vol. 1, (2002).

[13] D. C. Rapaport. The art of molecular dynamics simulation. Cambridge university press, (1995).

[14] K.A. Fichthorn and W.H. Weinberg. Theoritical foundations of dynamical monte carlo simulations. J, Chem. Phys., 95(2):1090-1096, (1991).

[15] P. E. Kloeden and E. Platen. Numerical solution of stochastic differential equations. Springer series in Applications of Mathematics, 23, (1999).

[16] W. E and B. Engquist. The hetrogeneous multi-scale methods. Comm. Math. Sci., 1:87-133, 2003.

[17] T. R. Waite. Theoritical treatment of the kinetics of diffusion-limited reactions. Physical Review, 107(2):463-470, (1957).

[18] M. V. Smoluchowski. Z. physik. Chem., 92:192, (1917).

[19] A. B. Bortz, M. H. Kalos, and J. L. Lebowitz. A new algorithm for monte carlo simulation of ising spin systems. J. Computational Physics, 17:10, (1975). 
[20] F. Graziani. Radiation transport in hetrogeneous materials. APS meeting : Conference on Computational Physics 2002, August 25-28(San Diego), (2002).

[21] H. Goldstein. Classical mechanics. Addison Wesley publishing company, (1971).

[22] C. Martin, M. Richou, W. Sakaily, B. Pégourié, C. Brosset, and P. Roubin. Multiscale study of the porosity of carbon deposits collected in Tore Supra. J. Nucl. Mater., 363-365:1251$1255,(2007)$.

[23] D. W. Brenner. Empirical potential for hydrocarbons for use in simulating the chemical vapour deposition of diamond films. Phys. Rev. B, 42:9458, (1990).

[24] K. Nordlund, J. Keinonen, and T. Mattila. Formation of ion irradiation induced small-scale defects on graphite surfaces. Phys. Rev. Lett., 77:699, (1996).

[25] H. J. C. Berendsen, J. P. M. Postma, W. F. Van Gunsteren, A. DiNola, and J. R. Haak. Molecular dynamics with coupling to an external bath. J. Chem. Phys., 81(8):3684-3690, (1984).

[26] Arthur F. Voter, Francesco Montalenti, and Timothy C. Germann. Extending the time scales in atomistic simulation of materials. Annu. Rev. Mater. Res., 32:321-346, (2002).

[27] J. M. Lahtinen. Diffusive dynamics of interacting particles in equilibrium and under hydrodynamic sedimentation. Ph.D Dissertation, Laboratory of Physics, Helsinki university of technology, Finland, (2002).

[28] K. Nordlund. Kinetic Monte Carlo Notes. http://beam.acclab.helsinki.fi/ knordlun/mc/.

[29] R. A. Causey, M. I. Baskes, and K. L. Wilson. The retention of deuterium and tritium in poco axf-5q graphite. J. Vac. Sci. Technol. A, 4(3):1189-1192, (1986). 
[30] G. Federici and C.H. Wu. Modelling of plasma hydrogen isotope behavious in porous materials (graphites/carbon-carbon composites). J. Nucl. Mater., 186:131-152, (1992).

[31] K. Ashida, K. Ichimura, M. Matsuyama, and K. Watanabe. Thermal desorption of hydrogen, deuterium and tritium from pyrolytic graphite. J. Nucl. Mater., 128 \& 129:792, (1984).

[32] H. Atsumi. Hydrogen retention in graphite and carbon materials under a fusion reactor enviornment. Jnl. Nucl. Mater., 313-316:543-547, (2003).

[33] A. A. Haasz, P. Franzen, J. W. Davis, S. Chiu, and C. S. Pitcher. Two-region model for hydrogen trapping in and release from graphite. J. Appl. Phys., 77(1):66-86, (1995).

[34] G. Duesing, H. Hemmerich, W. Sassin, and W. Schilling. Proceedings of the International Conference on Vacancies and Interstitials in Metals, Jülich, Germany, 1968. (North-Holland, Amsterdam, 1970), page 246, (1970).

[35] A. Rai, R. Schneider, M. Warrier, P. Roubin, C. Martin, and M. Richou. Kinetic monte-carlo modeling of hydrogen retention and re-emission from tore-supra deposits. J. Nucl. Mater., http://dx.doi.org/10.1016/j.jnucmat.2008.12.316, (2009).

[36] W. Eckstein. Computer simulations of ion-solid interactions. Springer series in material science 10, Springer-Verlag, (1991).

[37] W. Eckstein. Calculated sputtering, reflection and range values. Rep. IPP 9/132, MaxPlanck-Institut für Plasmaphysik, Garching, Germany, (2002).

[38] E. Tsitrone, D. Reiter, T. Loarer, C. Brosset, J. Bucalossi, L. Begrambekov, C. Grisolia, A. Grosman, J. Gunn, and et al. J. Hogan. Role of wall implantation of charge exchange neutrals in the deuterium retention for Tore Supra long discharges. J. Nucl. Mater., 337339:539-543, (2005). 
[39] P. Franzen and E. Vietzke. Atomic release of hydrogen from pure and boronized graphites. J. Vac. Sci. Technol. A, 12(3):820-825, (1994). 


\section{List of Figures}

1 Multi-scale schematic: The porous geometry creating ansatz on the bottom left side can be used to create both, granules separated by voids and crystallites separated by micro-voids; molecular dynamics (MD) is used to study hydrogen transport in crystallites; DiG_TGD is the version of the multi-scale code that handles trans-granular-diffusion (TGD) and DiG_Bulk is the version that handles bulk diffusion; Note that various parameters (explained in the text) are transferred from MD to DiG_TGD and from DiG_TGD to DiG_Bulk.

2 Porous Graphite structure created using the algorithm described in Section 3 .

3 Initial configuration of hydrogen in crystal graphite used in our MD simulations.

4 Interstitial trajectory at (a) $150 \mathrm{~K}$, (b) $900 \mathrm{~K}$. from MD.

$5 \quad$ Schematic of the macro-scale geometry.

6 Recombination rates from meso-scales as a function of temperatures for different incident fluxes.

$7 \quad$ The Bulk simulation model schematic. 
8 Macroscopic bulk diffusion scheme (Note that it takes $D_{T G D}$ as input from the simulations at the mesoscale).

9 Void size distribution for the macropores created from the simulation.

10 Retained amount of hydrogen for mesopores and macropores. The incident flux was $10^{20} \mathrm{H} / \mathrm{m}^{2} / \mathrm{s}$.

11 A view of the $\mathrm{X}-\mathrm{Y}$ plane located at $\mathrm{Z}=5.5 \times 10^{-8} \mathrm{~m}$. The white, grey and pale colored regions correspond to void, surface and the bulk of the graphite respectively. Open green circle: adsorbed hydrogen atom, red dots: trapped hydrogen atom, cyan dots: $H_{2}$ molecules in the crystallites, magenta: $H_{2}$ molecules in the void and blue dots: two trapped hydrogen atoms close enough to form a molecule.

12 Released amount of hydrogen atoms and molecules as a function of temperature for mesopores and macropores. 


\section{Multi-scale model}

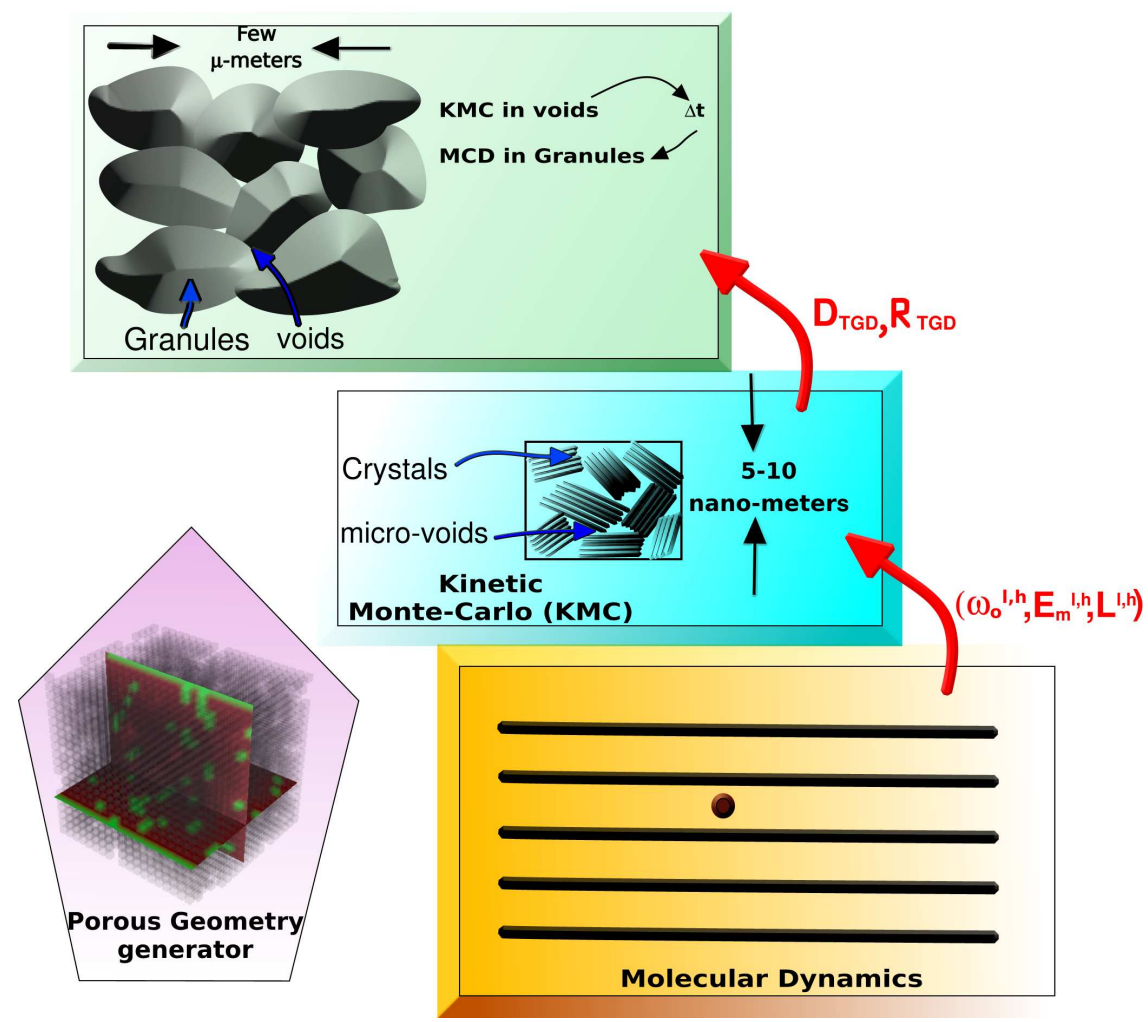

Fig. 1. Multi-scale schematic: The porous geometry creating ansatz on the bottom left side can be used to create both, granules separated by voids and crystallites separated by micro-voids; molecular dynamics (MD) is used to study hydrogen transport in crystallites; DiG_TGD is the version of the multi-scale code that handles trans-granular-diffusion (TGD) and DiG_Bulk is the version that handles bulk diffusion; Note that various parameters (explained in the text) are transferred from MD to DiG_TGD and from DiG_TGD to DiG_Bulk. 


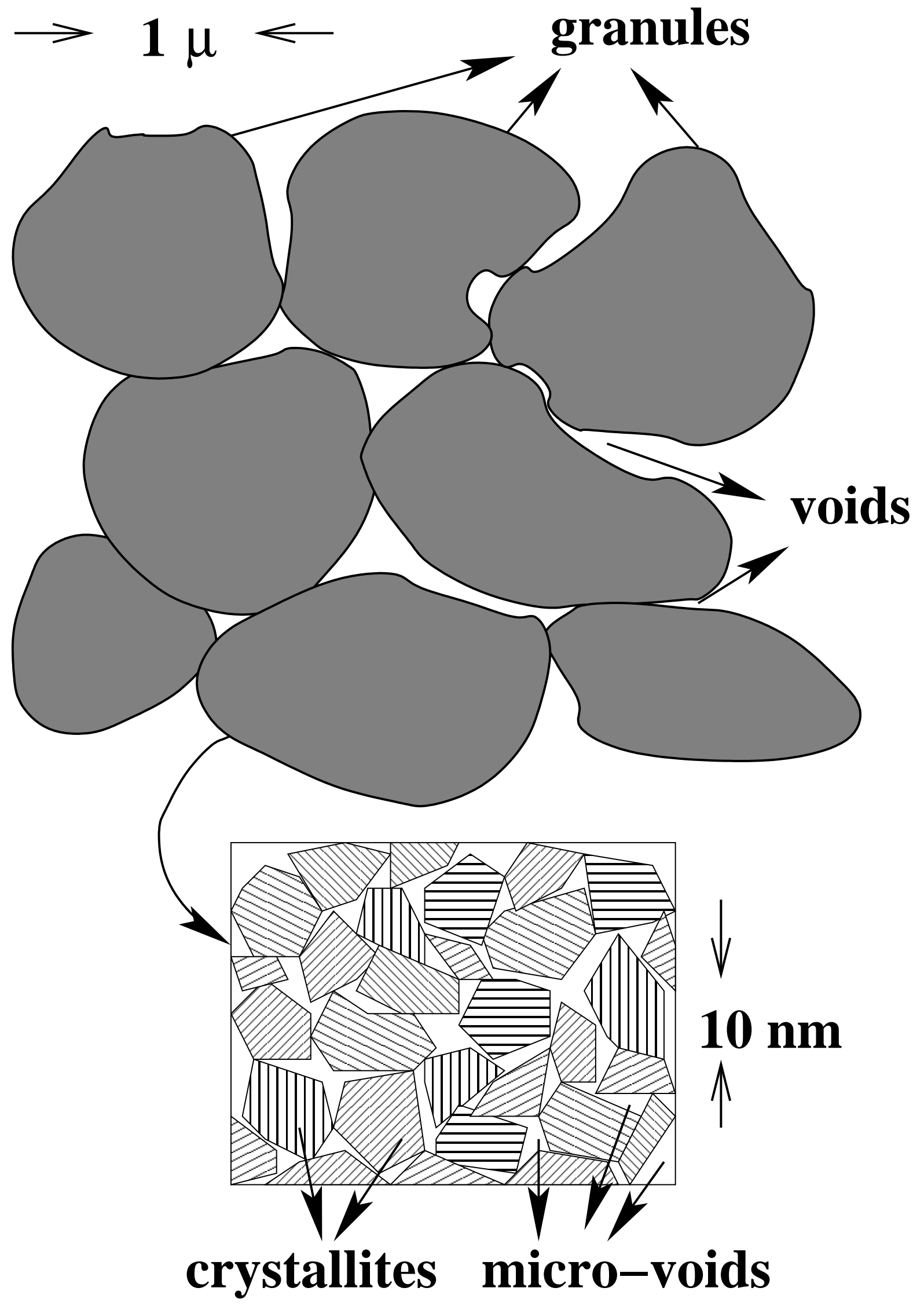

Fig. 2. Porous Graphite structure created using the algorithm described in Section 3. 


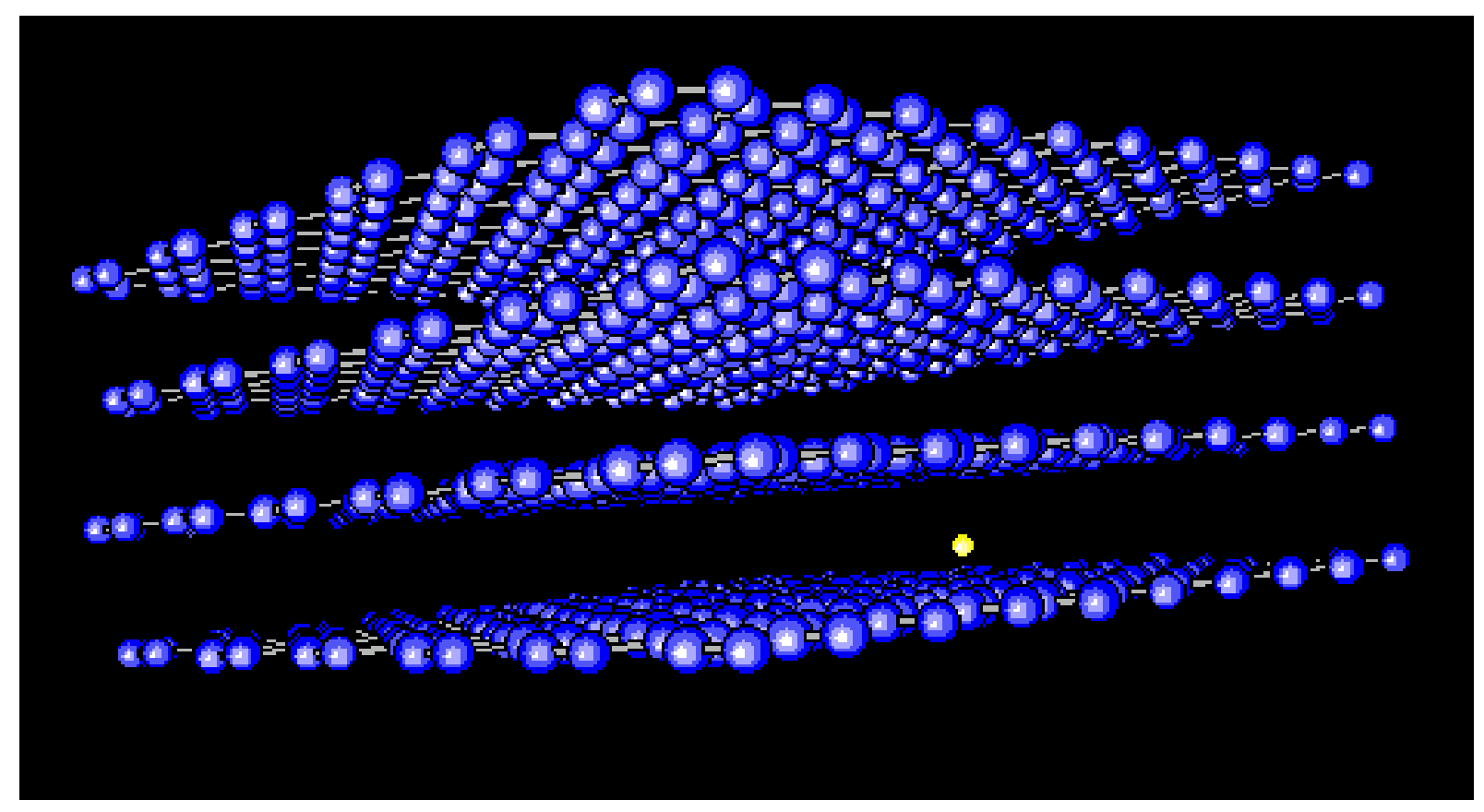

Fig. 3. Initial configuration of hydrogen in crystal graphite used in our MD simulations. 
(a)

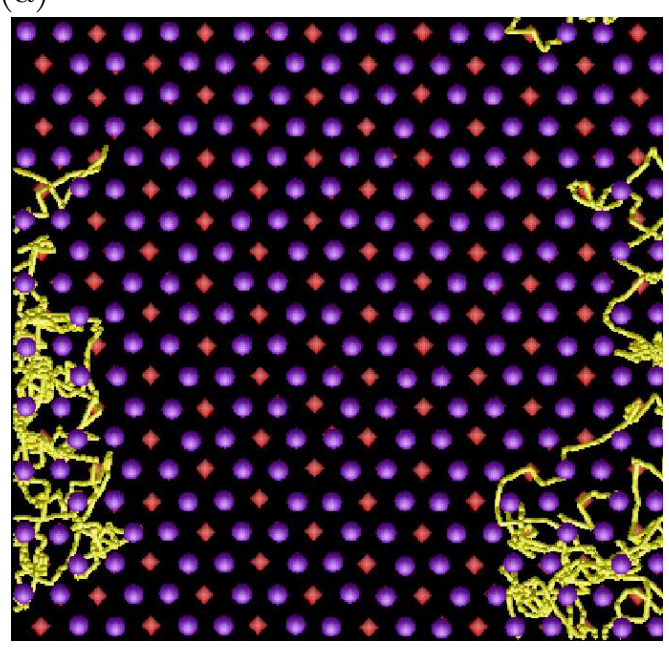

(b)

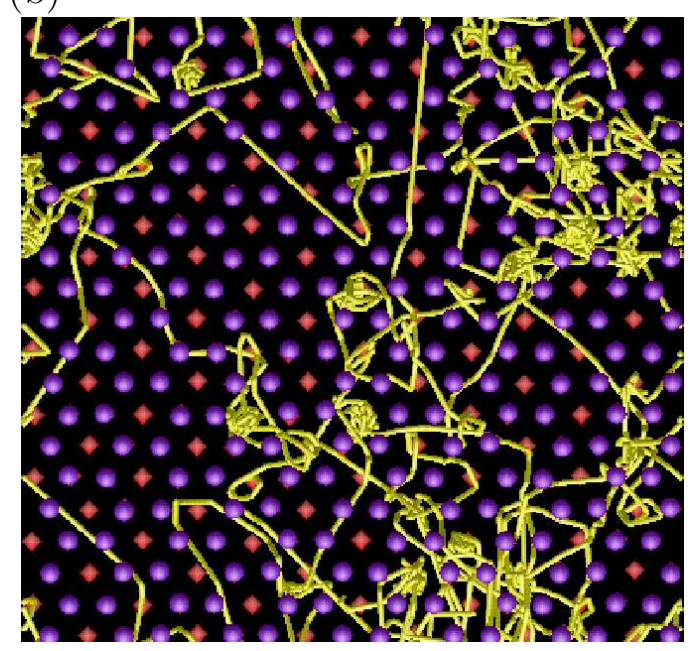

Fig. 4. Interstitial trajectory at (a) $150 \mathrm{~K}$, (b) $900 \mathrm{~K}$. from MD. 


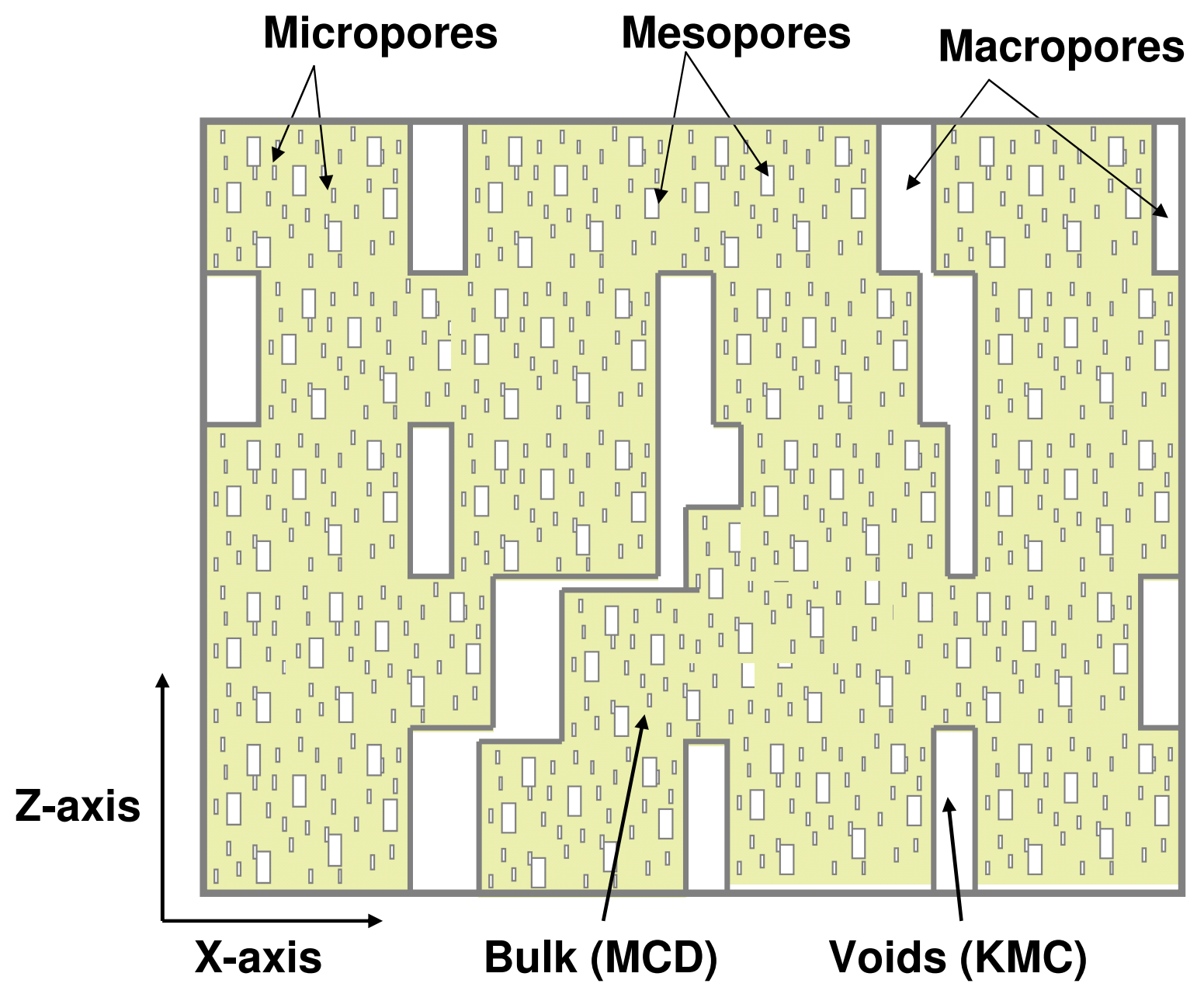

Fig. 5. Schematic of the macro-scale geometry. 


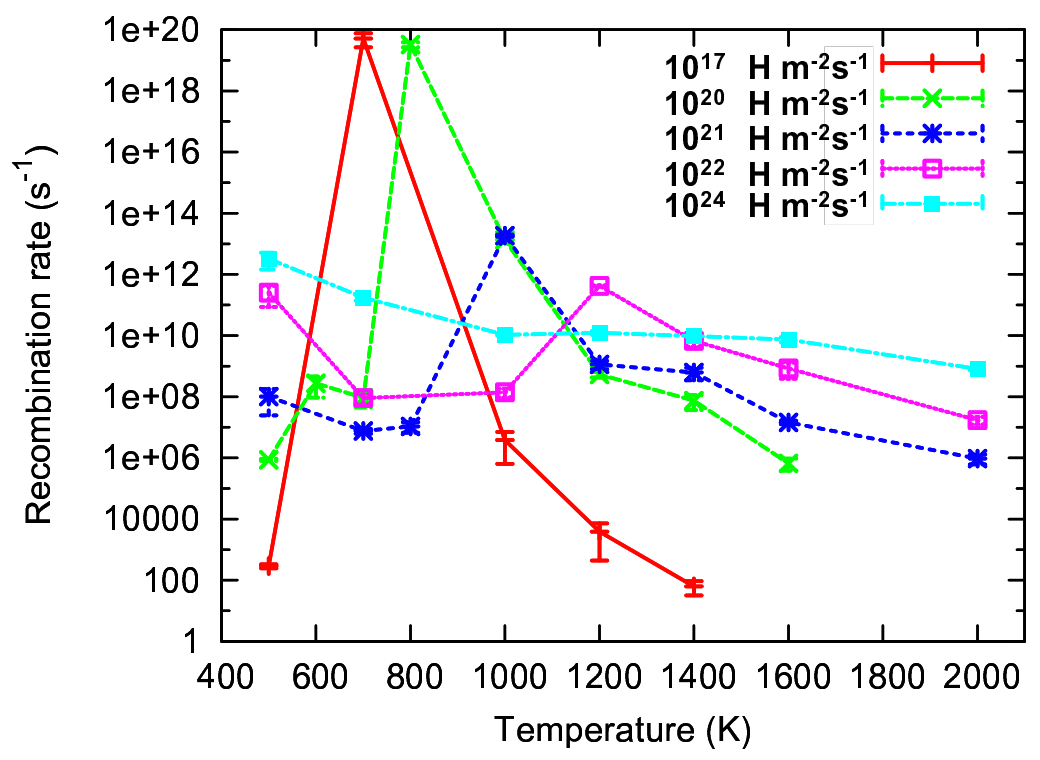

Fig. 6. Recombination rates from meso-scales as a function of temperatures for different incident fluxes. 


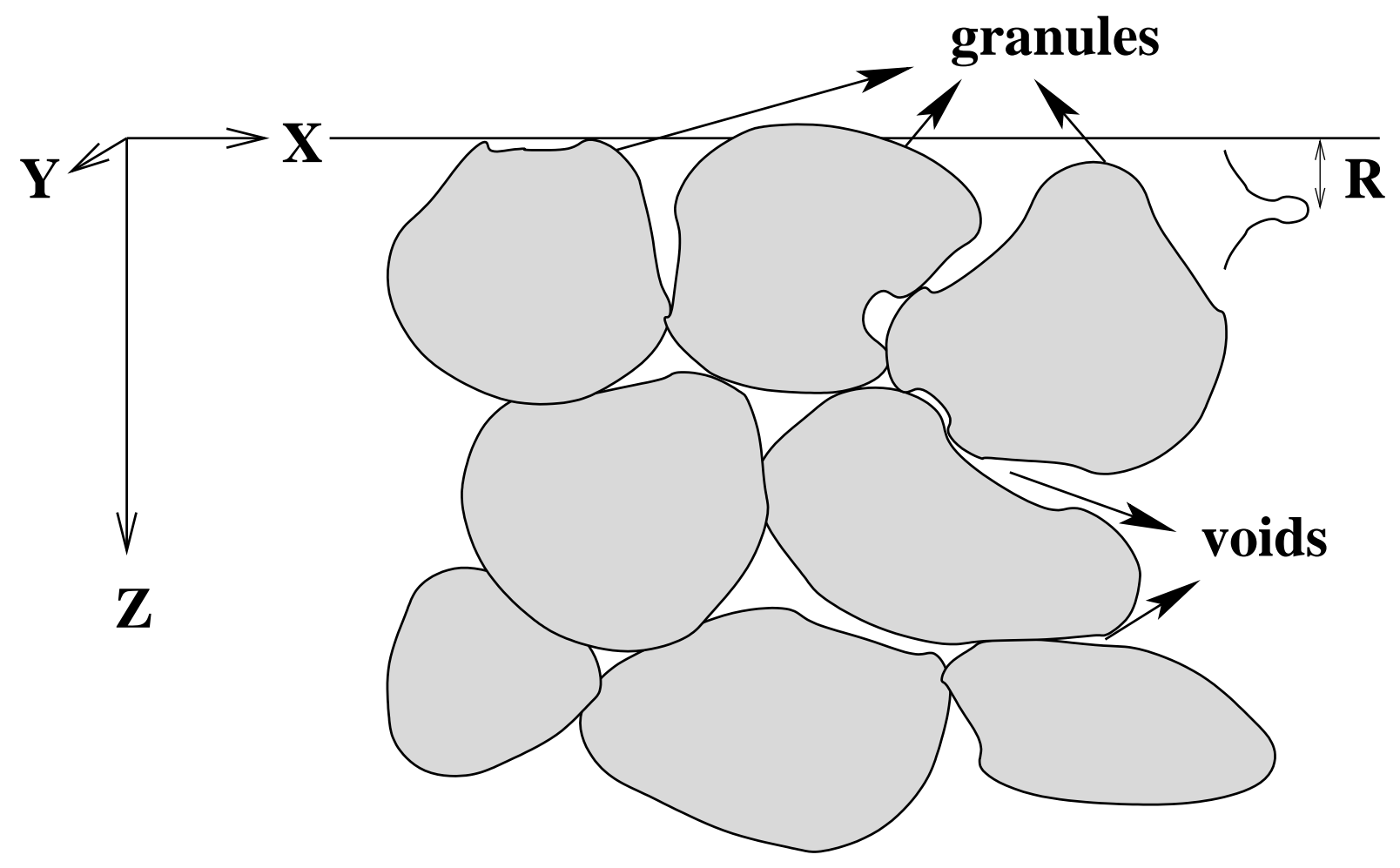

Fig. 7. The Bulk simulation model schematic. 


\section{Read inputs}

\section{Initialize Parameters}

Initialize graphite structure

Initialize particles

Loop for statistics

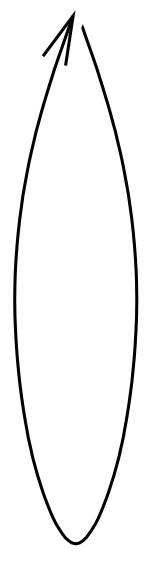

KMC for particles in the VGI

MCD for particles in the bulk ${ }^{\text {get } \Delta t}$

End loop

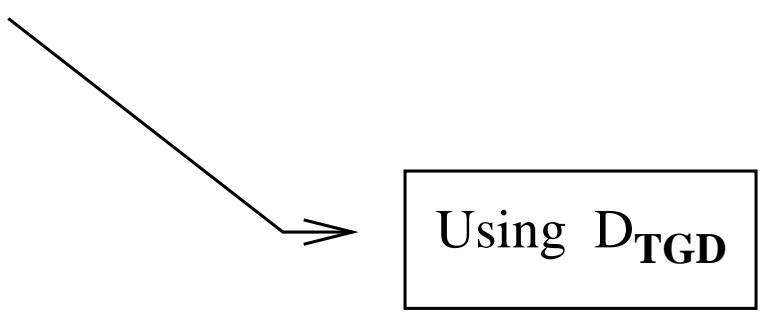

\section{Postprocessing}

Fig. 8. Macroscopic bulk diffusion scheme (Note that it takes $D_{T G D}$ as input from the simulations at the mesoscale). 


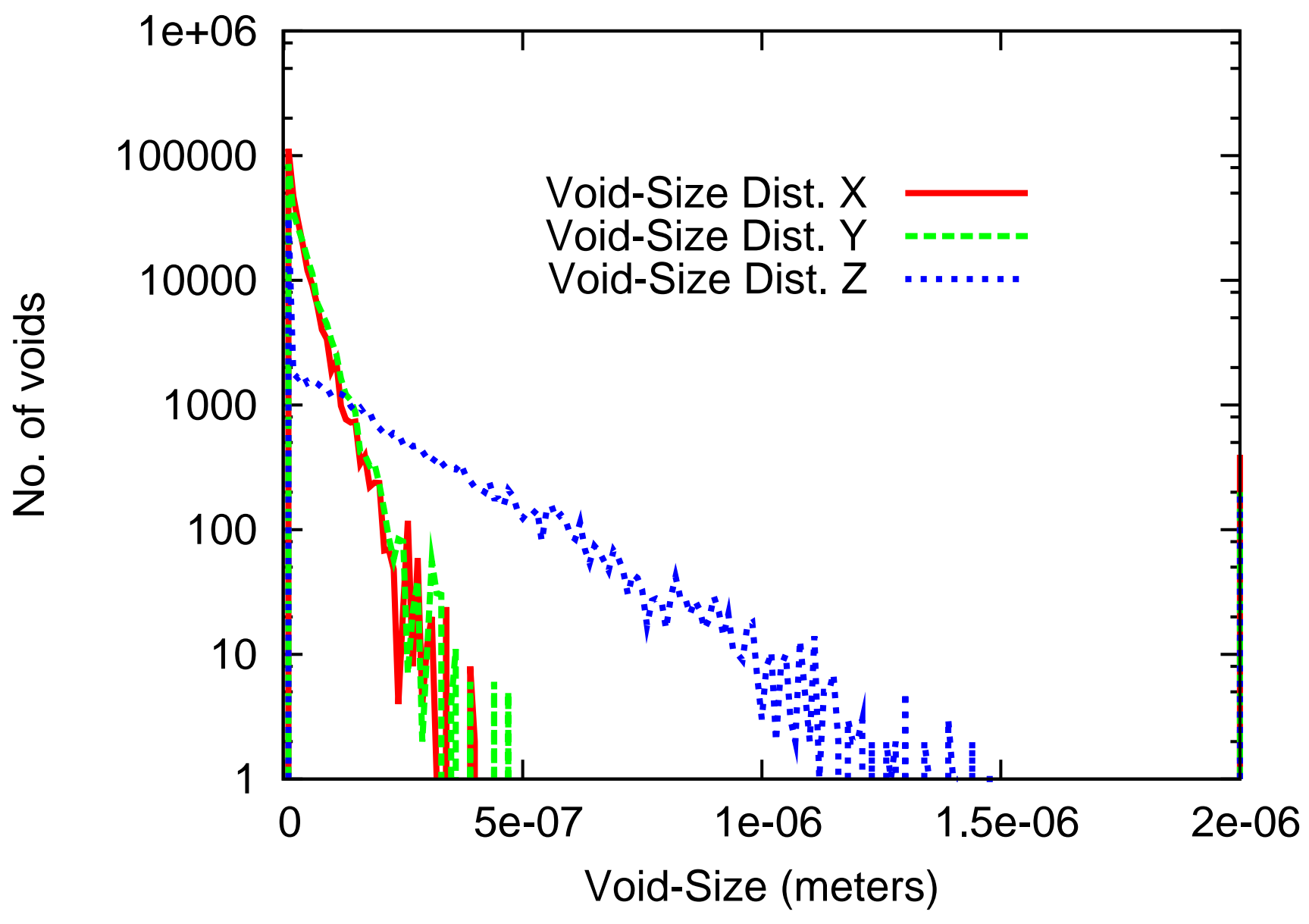

Fig. 9. Void size distribution for the macropores created from the simulation. 


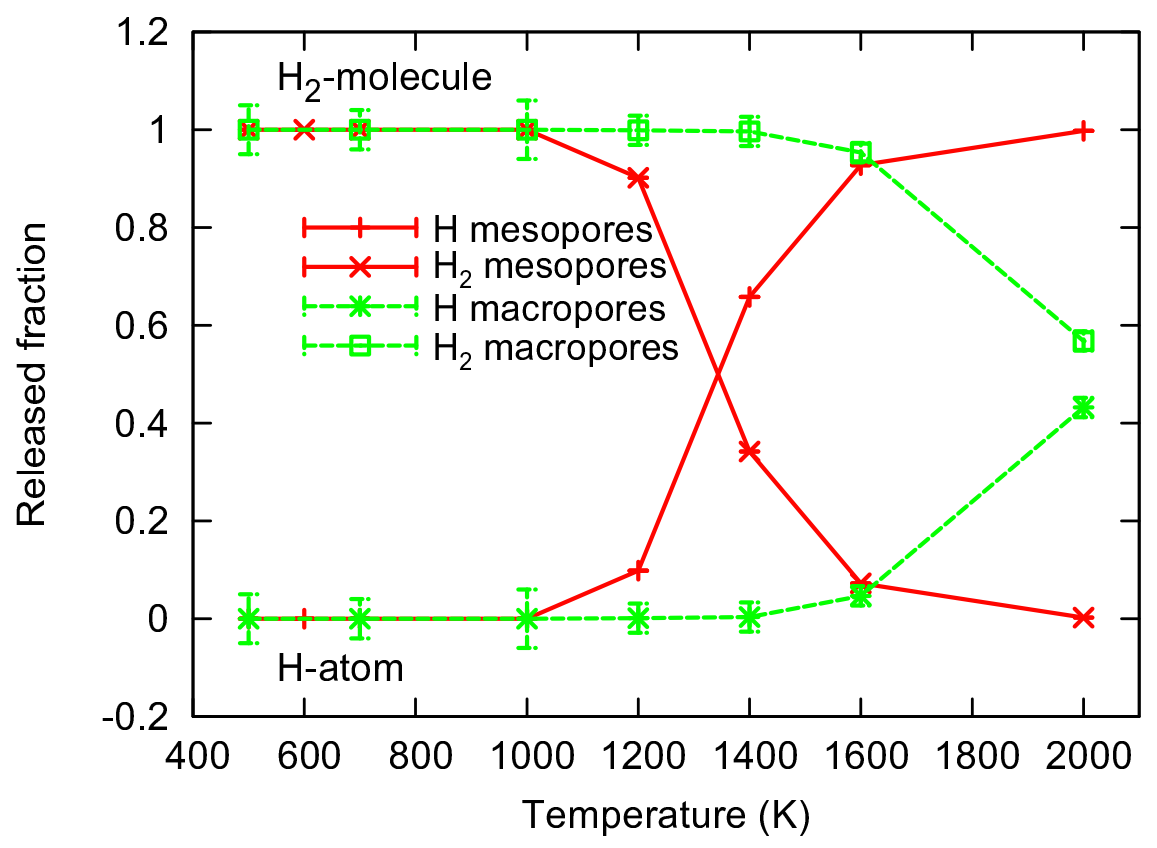

Fig. 10. Retained amount of hydrogen for mesopores and macropores. The incident flux was $10^{20} \mathrm{H} / \mathrm{m}^{2} / \mathrm{s}$. 


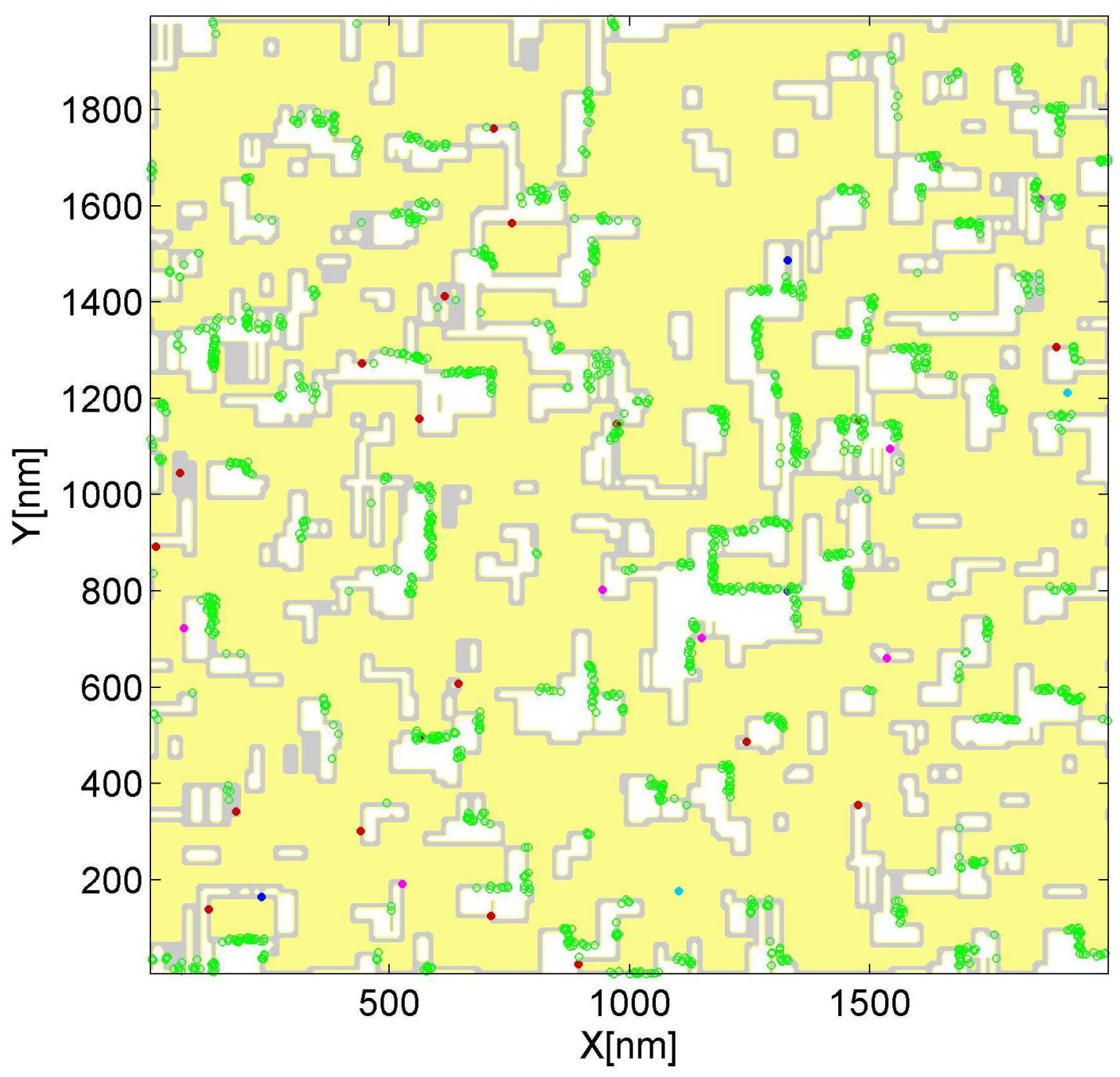

Fig. 11. A view of the $\mathrm{X}-\mathrm{Y}$ plane located at $\mathrm{Z}=5.5 \times 10^{-8} \mathrm{~m}$. The white, grey and pale colored regions correspond to void, surface and the bulk of the graphite respectively. Open green circle: adsorbed hydrogen atom, red dots: trapped hydrogen atom, cyan dots: $H_{2}$ molecules in the crystallites, magenta: $\mathrm{H}_{2}$ molecules in the void and blue dots: two trapped hydrogen atoms close enough to form a molecule. 


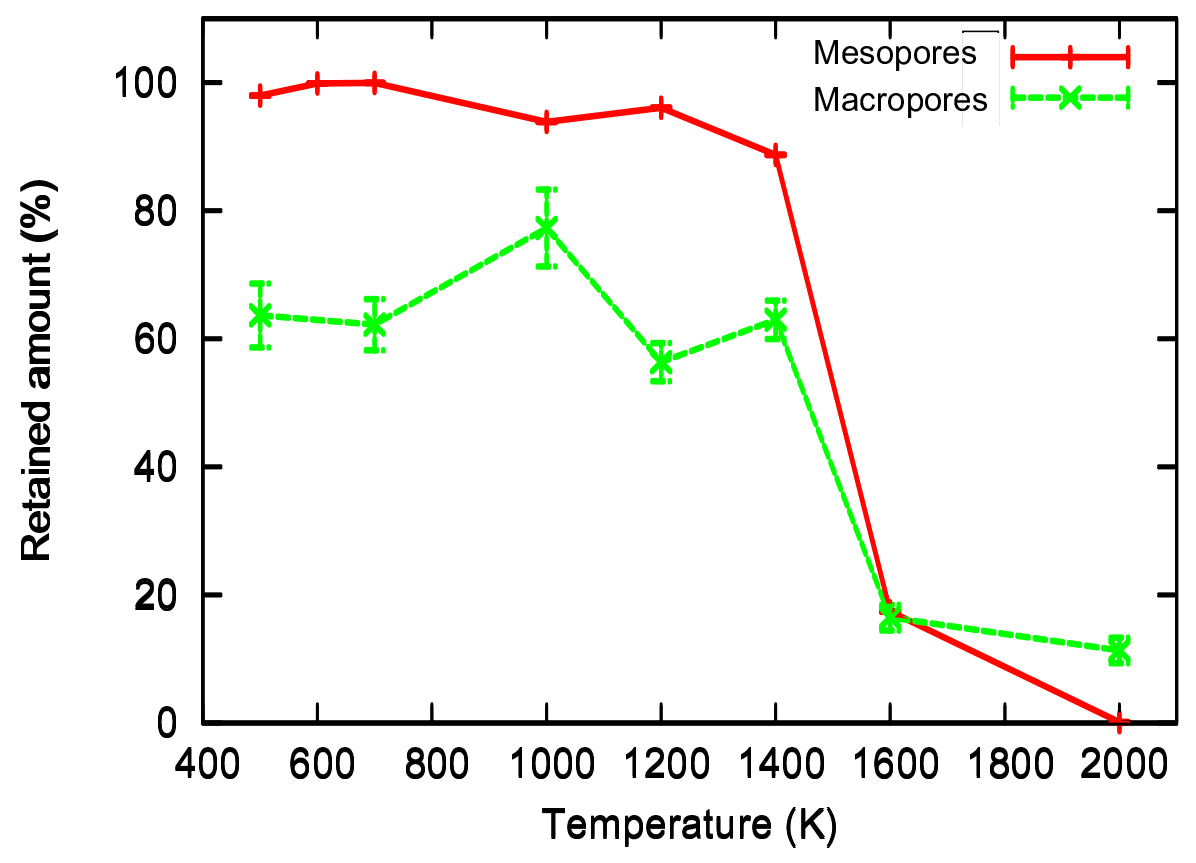

Fig. 12. Released amount of hydrogen atoms and molecules as a function of temperature for mesopores and macropores. 